\title{
Quantitative impact of Dixon $\mu$ map variability in dual-time-point brain PET/MR
}

\author{
Kimberly Jackson ${ }^{1 *}$, Rachel Bartlett ${ }^{1}$, Kent Friedman ${ }^{1}$, Timothy Shepherd ${ }^{1}$, Thomas Koesters ${ }^{1}$, Jose Teruel', \\ Mathias Fenchel ${ }^{2}$, Gerardo Hermosillova-Valadez ${ }^{2}$, David Faul ${ }^{1}$, Fernando Boada ${ }^{1}$ \\ From PSMR 2015: 4th Conference on PET/MR and SPECT/MR \\ La Biodola, Isola d'Elba, Italy. 17-21 May 2015
}

${ }^{1}$ Center for Biomedical Imaging, Department of Radiology, NYU Langone Medical Center, New York, USA
Dixon-based MR is acquired for attenuation correction of brain PET during PET/MR. Early adopters of PET/MR have noted variability in the performance of Dixon-based tissue segmentation, and questions exist regarding potential impacts on quantitative accuracy in dual-time-point studies. Ten patients injected with $10 \mathrm{mCi}$ FDG underwent dual-time-point clinical brain PET/MR on a Siemens mMR, with image reconstructions based on data acquired at $45-60$ and $75-90$ minutes. Dixon $\mu$ maps were obtained the time of FDG injection and at 75 minutes. $8 \mathrm{cc}$ of Gadavist was injected for post-contrast MR at 30 minutes. Subjects were removed from the table and repositioned prior to the 75 minute scan. The delayed $\mu$ map was registered to the original $\mu$ map using MIMneuro. The aligned image data was copied onto the original $\mu$ map DICOM file using Matlab. Early time-point PET data was reconstructed using both the early and delayed $\mu$ maps. Atlas-based segmentation was performed to compare regional SUV values. When comparing the delayed versus original $\mu$ map reconstructions, regional SUV values varied on average by $+1.9 \%$ for both SUVmax and SUVmean. For large brain structures, SUVmax and SUVmean varied by $-0.5 \%$ to $+5.6 \%$ and -0.2 to $+4.7 \%$, respectively. For deep brain structures, SUVmax using the delayed reconstruction varied by $-0.5 \%$ to $+3.7 \%$ and SUVmean varied by $-0.3 \%$ to $+3.6 \%$. Most differences in SUV were higher when using the delayed $\mu$ map. There is variability in regional SUV values for brain PET data reconstructed using two different aligned Dixon acquisitions performed on subjects undergoing repeat same-day PET/MR imaging, with SUV values $\sim 2 \%$ higher using the delayed $\mu$ map. This variability may impact results in dual-time-point brain PET/MR.

Authors' details

${ }^{1}$ Center for Biomedical Imaging, Department of Radiology, NYU Langone Medical Center, New York, USA. ${ }^{2}$ Siemens Healthcare, USA.

Published: 18 May 2015

doi:10.1186/2197-7364-2-S1-A74

Cite this article as: Jackson et al:: Quantitative impact of Dixon $\mu$ map variability in dual-time-point brain PET/MR. EJNMMI Physics 2015 2(Suppl 1):A74.

\section{SpringerOpen ${ }^{\circ}$}

(C) 2015 Jackson et al; licensee Springer. This is an Open Access article distributed under the terms of the Creative Commons Attribution License (http://creativecommons.org/licenses/by/4.0), which permits unrestricted use, distribution, and reproduction in any medium, provided the original work is properly cited. 